\title{
Reactivation of Motor Brain Areas during Explicit Memory for Actions
}

\author{
Lars Nyberg,* Karl Magnus Petersson, † Lars-Göran Nilsson,‡ J ohan Sandblom, $†$ \\ Carola Åberg, $\ddagger$ and Martin Ingvar† \\ *Department of Psychol ogy, Umeå University, Sweden; †Cognitive Neurophysiology Research Group, Department of Clinical Neuroscience, \\ Karolinska Institutet, Sweden; and łDepartment of Psychology, Stockholm University, Sweden
}

Received September 20, 2000; published online May 30, 2001

Recent functional brain imaging studies have shown that sensory-specific brain regions that are activated during perception/encoding of sensory-specific information are reactivated during memory retrieval of the same information. Here we used PET to examine whether verbal retrieval of action phrases is associated with reactivation of motor brain regions if the actions were overtly or covertly performed during encoding. Compared to a verbal condition, encoding by means of overt as well as covert activity was associated with differential activity in regions in contralateral somatosensory and motor cortex. Several of these regions were reactivated during retrieval. Common to both the overt and covert conditions was reactivation of regions in left ventral motor cortex and left inferior parietal cortex. A direct comparison of the overt and covert activity conditions showed that activation and reactivation of left dorsal parietal cortex and right cerebellum was specific to the overt condition. These results support the reactivation hypothesis by showing that verbal-explicit memory of actions involves areas that are engaged during overt and covert motor activity. 2001 Academic Press

\section{INTRODUCTION}

Functional brain imaging techniques such as PET and $\mathrm{fMRI}$ offer ways of separately studying the brain systems that mediate fundamental memory processes like encoding (acquisition) and retrieval. Incidental as well as intentional encoding of episodic information have been associated with increased activity in left prefrontal cortex and in medial temporal lobe regions, whereas explicit episodic retrieval processes have been associated with increased activity in other regions, including right prefrontal and medial parietal regions (see Cabeza and Nyberg, 2000).

Recently, similarities in activation patterns for encoding and retrieval have also been investigated with functional brain imaging. Cognitive (e.g., Morris et al., 1977) as well as neuronal (e.g., Alvarez and Squire, 1994) models predict that similar brain regions should be engaged during encoding (memory formation) and retrieval. This prediction is supported by findings from several independent studies. Persson and Nyberg (2000) contrasted encoding of the spatial location of visual words with encoding conditions that did not involve encoding of spatial location and retrieval of spatial information with retrieval conditions that did not involve spatial retrieval. A conjunction analysis (Price and Friston, 1997) revealed that increased activity in bilateral inferior parietal cortex (Brodmann area 39/40) was common to the encoding and retrieval contrasts. Hence, this study showed that portions of the dorsal where pathway are activated during both encoding and retrieval of spatial event information (cf., Köhler et al., 1998). Wheeler et al. (2000) observed that regions in visual cortex were differentially activated during viewing of pictures and during retrieval of pictorial information based on verbal labels. In addition, Wheeler et al. found activation in overlapping parts of auditory cortex during perception and retrieval of auditory information. Similarly, Nyberg et al. (2000) found that auditory responsive cortex in the temporal lobes was activated during both encoding and retrieval of sound information. Activation of auditory responsive cortex during retrieval was observed when subjects intentionally attempted to retrieve auditory information in response to a visual word cue that had been paired with a sound at encoding. Following word-sound pairing at encoding, auditory activation was also observed during simple visual word recognition, i.e., when there was no demand to retrieve auditory information. The latter observation indicates that reactivation of some of the encoding activity at retrieval is not simply a function of selective attention but a genuine retrieval effect.

Taken together, the above mentioned studies suggest that sensory-specific brain regions are activated during encoding and retrieval of sensory-specific information. In the present study we sought to further examine this notion by investigating encoding and re trieval of enacted events. Specifically, we were interested in examining whether verbal retrieval of action 
phrases (e.g., roll the ball) would be associated with activation of motor brain regions if the actions had actually been performed during encoding.

It is well known that retrieval is higher following enacted encoding compared to retrieval following a standard verbal encoding condition (for a review, see Engelkamp, 1998). This is true for children (Cohen and Stewart, 1982), adults (Nyberg and Nilsson, 1995), and elderly (Bäckman and Nilsson, 1984), as well as for various patient populations, including demented patients (Karlsson et al., 1989) and patients with frontal lobe dysfunction (McAndrews and Milner, 1991; Mimura et al., 1998). It has been proposed that the superior retrieval performance following enacted encoding is, at least in part, due to the possibility to base retrieval on motor information following enacted encoding. Support for this position was provided in an event-related-potential (ERP) study in which ERPs were recorded during retrieval (recognition) following enacted and verbal encoding (Heil et al., 1999). Retrieval following enacted encoding was associated with a larger frontocentral negativity, which was taken as evidence that the superior memory following enacted encoding was due to reactivation of motor information. A recent PET study (Nilsson et al., 2000) also provided evidence that motor brain regions are activated during retrieval of enacted events. In the Nilsson et al. study, differential activation was observed in right motor cortex when retrieval following enacted encoding was contrasted with retrieval following verbal encoding.

Together, the results from the ERP study by Heil and colleagues and the PET study by Nilsson and colleagues suggest that verbal retrieval is associated with activation of motor brain regions following enacted encoding. However, as none of these studies included measurement of brain activity during actual encoding, it cannot be concluded that those motor areas that were active during encoding were subsequently reactivated during retrieval. To directly address this issue, the present study included measurement of brain activity with PET during encoding as well as retrieval of enacted and verbal events. A condition involving imaginary motor activity during encoding was also induded. There is substantial evidence for similarities in brain activation patterns during imagined and real execution (Crammond, 1997; Lotze et al., 1999), but regional differences have also been observed (e.g., Lotze et al., 1999). Of main concern here was to compare the brain activation pattern during retrieval following real versus imagined motor activity during encoding.

\section{METHODS}

\section{Subjects}

Ten normal healthy right-handed men (handedness was defined by selfassessment in a semistructured interview) were included in the study (mean age $=27.1$ years, SD $=4.12$ years; range $=22-37$ ). All subjects had high-school (gymnasium) level education or higher. The subjects were prescreened and none used any medication, had a history of drug use (including current smoking), head trauma, neurological or psychiatric illness, or family history of neurological or psychiatric illness. The study was approved by the local Ethics and Radiation Safety Committees at the Karolinska Hospital. Informed consent was given by all subjects.

\section{Experimental Design}

The experiment was set up as a categorical design including 6 different conditions, all repeated twice in two cyclically permuted blocks. To minimize motor imagery processes during the verbal condition, the verbal condition was presented first followed by a counterbalanced order of the overt and covert enactment conditions (i.e., verbal encoding, cued recall, verbal encoding, cued recall, imagery encoding, cued recall, enactment encoding, cued recall ....). It cannot be ruled out that this way of presenting the experimental conditions caused order effects that affect the contrasts involving the verbal condition. It should be stressed, though, that the overt and covert conditions were fully counterbalanced. M easurements were made on all subjects in all conditions.

\section{Tasks}

The experiment involved six conditions, and each condition was performed (and scanned) twice. There were three encoding conditions. During each encoding condition, 16 unique verb-noun commands were read by a female experimenter at the rate of $6.5 \mathrm{~s} / \mathrm{item}$ (nine of these were presented during the 60-s scan window). In the verbal encoding condition, subjects were instructed to silently rehearse each command until the next item was presented and so on until the entire list had been presented. In the enactment encoding condition, subjects were instructed to overtly perform the activity described by each command (with an imaginary object). This was done with their right arm/hand while lying down on the scanner table. The items were constructed such that they were possible to perform with one arm/hand when lying down. Some items mainly involved hand movements (e.g., "make a fist"), but most items additionally involved arm and shoulder movements (e.g., "look at your hand"). I n the imaginary encoding condition, subjects covertly performed the activities described by each command. The lists were counterbalanced across conditions over subjects.

About 9.5 min after each encoding condition, a retrieval condition followed. In all retrieval conditions, the experimenter read 16 verbs at the rate of $5 \mathrm{~s} /$ item (12 of these were presented during the 60-s scan window). Subjects were instructed to generate, for each 
verb, the noun that had been paired with the verb during encoding. When they could not retrieve a noun, they said "no" (in Swedish). The verbs were presented in a different order at retrieval than at encoding.

\section{PET Scanning Procedure and Data Analysis}

Each subject underwent 12 measurements of regional cerebral blood flow with a 3-D ECAT EXACT HR PET scanner (Wienhard et al., 1994) and bolus injections of [0-15]water (12-14 mCi per scan). Subjects were blindfolded during all scans. The PET scanner was used in 3-D sampling mode producing 60-s tracer uptake images. The different conditions were initiated at the time of tracer injection and the scanning was automatically started when the brain radioactivity exceeded a predetermined level of radioactivity above background. Scatter correction was made and a 2-D transmission scan was used for attenuation correction. One subject had to temporarily leave the scanner bed so a second transmission scan was acquired after the last activation scan. Normalized integrated regional counts were used as an index of regional cerebral blood flow because such counts are linearly correlated with blood flow (Hersovitch et al., 1983).

Using SPM99 (http://www.fil.ion.bpmf.ac.uk), the PET images were realigned, anatomically normalized, and transformed into a common stereotactic space as defined by the SPM99 template, an approximate Talairach space (Talairach and Tournoux, 1988), 3-D isotropic Gaussian filtered (14 mm FWHM), proportionally scaled to account for global confounders, and statistically analyzed. To localize activations, the SPM coordinates were converted to Talairach space using a nonlinear function (http://www.mrc-cbu.cam.ac.uk/ I maging/mnispace.html) and matched against the Talairach brain atlas. Activations were also plotted on mri templates to help determining their location. The general linear model was used to model the regional cerebral blood flow response data, and relevant contrasts corresponding to null-hypotheses were used to generate statistic images:

First, to identify brain regions differentially activated during encoding or retrieval, encoding and retrieval were directly contrasted with each other across type of condition (verbal, enactment, imaginary) (threshold $=\mathrm{P}<0.01$ corrected).

Second, to identify brain regions that were differentially activated during encoding of overtly enacted events, the encoding condition involving overt enactment was contrasted with (a) the verbal encoding condition and (b) the encoding condition involving covert enactment. To identify brain regions that were differentially active during covert encoding enactment, the encoding condition involving covert enactment was contrasted with the verbal encoding condition (threshold for all encoding contrasts $=\mathrm{P}<0.01$ corrected).
TABLE 1

Cued-Recall Performance (Proportion Correct) as Function of Experimental Condition

\begin{tabular}{lcc}
\hline \multicolumn{1}{c}{ Condition } & Mean & Standard deviation \\
\hline Overt enactment & 0.51 & 0.13 \\
Imaginary enactment & 0.39 & 0.08 \\
Verbal & 0.34 & 0.12 \\
\hline
\end{tabular}

Third, to examine whether retrieval following overt and covert encoding enactment was associated with increased activity in some of the brain regions that were engaged during encoding, the encoding activation maps were used as masks for the retrieval contrasts. Specifically, three masked analyses were conducted:

(1) (overt encoding enactment-verbal encoding) as mask for (retrieval following overt encoding enactment-retrieval following verbal encoding);

(2) (overt encoding enactment-covert encoding enactment) as mask for (retrieval following overt encoding enactment-retrieval following covert encoding enactment);

(3) (covert encoding enactment-verbal encoding) as mask for (retrieval following covert encoding enactment-retrieval following verbal encoding).

In this way, the analyses of retrieval activity were restricted to those regions that showed differential activation during encoding. The mask was thresholded at a voxelwise false positive rate of $\mathrm{P}<0.001$; df $=[1$, 105], and retrieval activations were considered significant if the t value was $>3.50$ (a voxel wise false positive rate of $\mathrm{P}<0.001 ; \mathrm{df}=[1,105])$.

\section{RESULTS}

\section{Memory Performance}

Table 1 shows retrieval performance as a function of experimental condition. As can be seen, in line with previous studies (e.g., Nyberg and Nilsson, 1995), retrieval was higher following encoding enactment than following verbal encoding [paired t tests, $P<0.01$ ] Performance following imaginary encoding enactment was intermediate and significantly different from the real enactment condition [P $<0.01]$. The difference in memory performance between imaginary enactment and verbal encoding was in the expected direction, but did not reach significance $[\mathrm{P}<0.10]$.

\section{Encoding-Retrieval Differences}

The contrast between encoding and retrieval re vealed differential activation during encoding and re trieval. Activated regions $(P<0.01$ corrected for multiple comparisons) during encoding included bilateral 
a
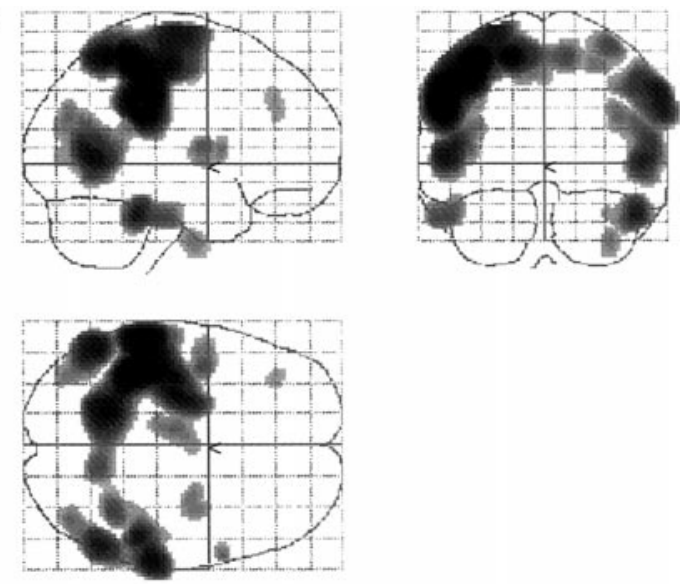

b
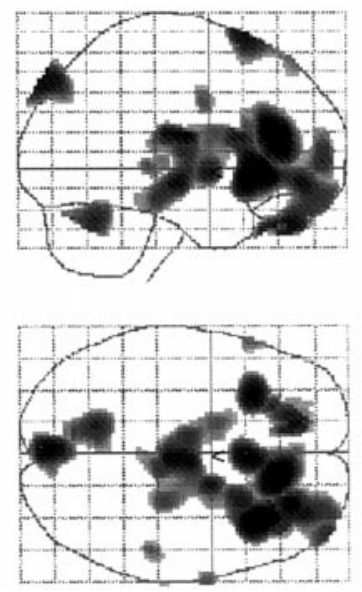

FIG. 1. Sagittal, coronal, and horizontal views of brain regions associated with encoding or retrieval. (a) Differential activation during encoding compared to retrieval. (b) Differential activation during retrieval compared to encoding (threshold in $a \& b=P<0.01$ corrected).

parietal cortex $[\mathrm{x}, \mathrm{y}, \mathrm{z}$ (Talairach and Tournoux, 1988) $=-55,-34,29, Z=$ Inf.; $63,-29,35, Z=7.79$, bilateral inferior temporal cortex $[x, y, z=-51,-30$, $-22, Z=6.11 ; 50,-38,-22, Z=7.43]$, left middle temporal cortex $[x, y, z=-53,-58,7, z=7.70]$, and left middle frontal gyrus $[x, y, z=-36,36,30, z=$ 4.94] (Fig. 1a). Activated regions $(P<0.01$ corrected for multiple comparisons) during retrieval included anterior cingulate gyrus $[x, y, z=14,34,17, z=7.78]$, bilateral frontal operculum $[x, y, z=36,23,-5, z=$ $7.34 ;-30,21,-4, z=7.31]$, precuneus $[x, y, z=-2$, $-81,46, Z=6.82]$, left cerebellum $[x, y, z=-14,-59$, $-21, z=6.02]$, and right frontopolar cortex $[x, y, z=$ 32, 48, $-9, Z=6.15$ ] (Fig. 1b).

\section{Encoding-Retrieval Similarities}

Figure 2a shows brain regions that were differentially active $(P<0.01$ corrected for multiple comparisons) when the encoding enactment condition was contrasted with the verbal encoding condition. These regions included left motor/premotor cortex $[x, y, z=$ $-26,-12,69, z=$ Inf.; $x, y, z=-46,3,15, z=7.13]$, left supplementary motor cortex $[x, y, z=-2,-7,46$, $Z=6.35]$, right midcingulate $[x, y, z=14,2,46, Z=$ 6.94], left somatosensory/motor cortex $[x, y, z=-36$, $-19,45, \mathrm{Z}=\mathrm{Inf}$.], and right cerebellum $[\mathrm{x}, \mathrm{y}, \mathrm{z}=18$, $-50,-18, Z=7.78]$. As shown in Fig. $2 b$, a subset of the regions that were activated at encoding was also activated at retrieval. This included regions in motor/ premotor cortex (Table 2). For one of these motor regions, the activation at encoding $(x, y, z=-46,3,15)$ overlapped with the activation at retrieval $(x, y, z=$ $-53,8,9)$. For the other region, the encoding peak was more dorsal $(x, y, z,=-26,-12,69)$ than the retrieval peak $(x, y, z=-49,-12,28)$. However, the activation at encoding was extensive and included significant ac- a
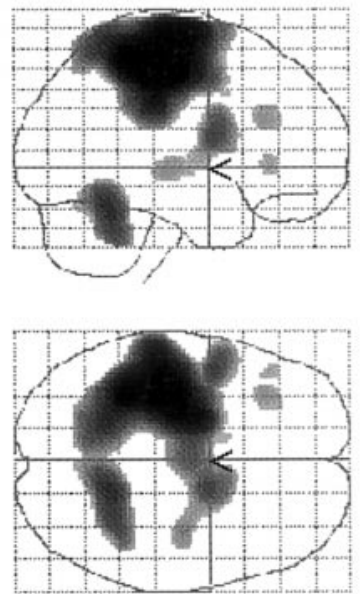

b
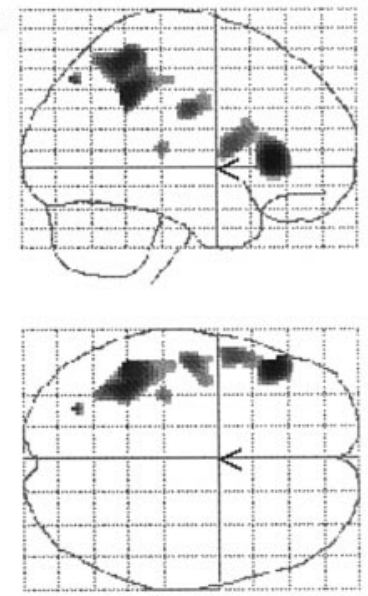
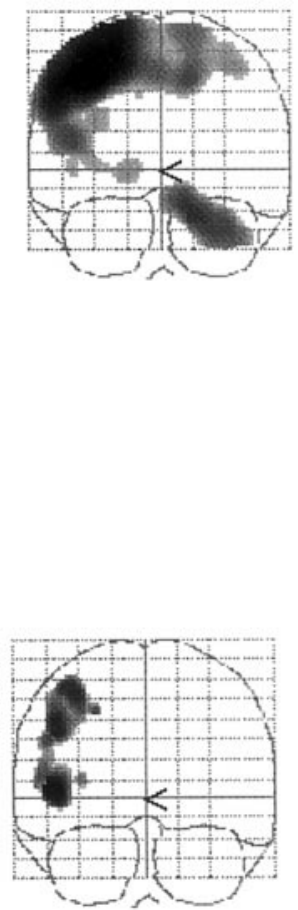

FIG. 2. Sagittal, coronal, and horizontal views of brain regions associated with encoding and retrieval of overt actions. (a) Differential activation during overt encoding enactment compared to verbal encoding. (b) Differential activation during retrieval following overt encoding enactment compared to retrieval following verbal encoding. The retrieval contrast was masked by the encoding contrast. The threshold for plotting activations in (a) was $\mathrm{P}<0.05$ corrected; the threshold for plotting activations in (b) was $\mathrm{P}<0.0005$ uncorrected (see text and Table 2 for inferential statistics). 
TABLE 2

Regions Showing Differential Activation during Retrieval

\section{Region}

Real enactment-verbal

Left inferior frontal cortex

Left motor/somatosensory cortex

Left premotor cortex

Left inferior parietal cortex

Left inferior parietal cortex

Left superior parietal cortex

Left auditory cortex

I maginary enactment-verbal

Left inferior parietal cortex

Left premotor cortex

Right somatosensory/motor cortex

Left motor/somatosensory cortex

Real enactment-imaginary enactment

Left inferior/superior parietal cortex

Right cerebellum

threshold of $Z=3.50$.

tivity at the retrieval peak ( $P<0.01$ corrected). This indicated that overlapping regions in motor cortex were activated at both encoding and retrieval.

Figure $3 a$ shows brain regions that were differentially active when the encoding condition involving imaginary enactment was contrasted with the verbal encoding condition. The activation pattern largely overlapped with that identified for the overt encoding condition (Fig. 2a), with the notable exception for cerebellar activation. Differentially activated regions ( $P<$ 0.01 corrected for multiple comparisons) included left motor/premotor cortex $[x, y, z=-30,-5,45, z=6.32$; $x, y, z=-22,-5,57, z=5.34]$, right midcingulate [ $x$, $y, z=16,2,40, z=5.63]$, and left somatosensory cortex [ $x, y, z=-53,-24,32, z=5.37]$. In fig. 3b, the corresponding retrieval activations are shown. These included regions in or near motor cortex (Table 2). Again, the location of the peaks in/near left motor cortex at retrieval $(x, y, z=-50,-12,26 ;-24,4,42)$ was more ventral than that for the encoding peaks $(x$, $y, z=-30,-5,45 ;-22,-5,57)$, but the encoding activation pattern included increased activity at both the more lateral ( $P<0.001$ uncorrected) and the more medial $(P<0.05$ corrected) retrieval peak.

Figure $4 a$ shows brain regions that were differentially active ( $P<0.01$ corrected for multiple comparisons) when the encoding condition involving real enactment was contrasted with the encoding condition involving imaginary enactment. The activation pattern was similar to that obtained when the real enactment encoding condition was contrasted with the verbal encoding condition (Fig. 2a), and included left motor/ premotor cortex [x, y, z $=-28,-14,69, \mathrm{z}=$ Inf.], left somatosensory cortex $[x, y, z=-36,-28,57, z=I n f$;
$-48,-20,47, Z=$ Inf.], left midbrain $[-14,-18,-6$, $Z=6.14]$, and right cerebellum $[x, y, z=20,-48,-20$, $Z=$ Inf.]. Of these regions, only two showed differential activation during retrieval; namely left parietal cortex and right cerebellum (Fig. 4b; Table 2).

\section{DISCUSSION}

Encoding and retrieval were found to be associated with distinct activity patterns. Encoding was associated with differential activity in bilateral temporo-parietal cortex and left middle frontal gyrus, whereas retrieval was associated with differential activity in the anterior cingulate gyrus, precuneus, cerebellum, bilateral frontal operculum, and right fronto-polar cortex. It should be noted that since the finding of distinct activity patterns during encoding and retrieval was generated from direct contrasts between encoding and retrieval scans, it cannot be ruled out that what now appears as activations during encoding was in fact deactivations during retrieval and vice versa. However, the regions that were observed in this study have been associated with encoding and retrieval in several previous studies in which different reference conditions have been used (see Cabeza and Nyberg, 2000). Thus, together with previous related findings, the present data suggests that different neural systems are operating during memory encoding and retrieval.

The analyses of encoding-retrieval overlap provided support that encoding and retrieval engage overlapping regions as well. This is consistent with recent findings of reactivation of visual and auditory brain regions during retrieval of visual or auditory information (Nyberg et al., 2000; Wheeler et al., 2000). More 
a
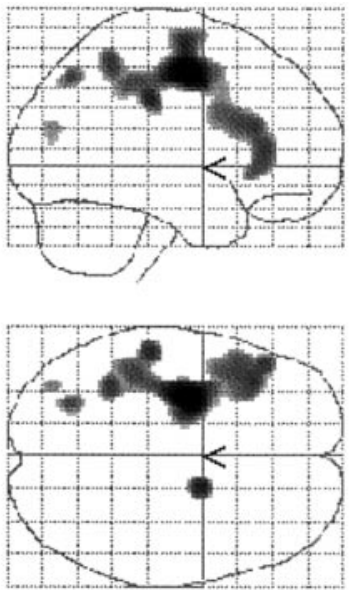

b

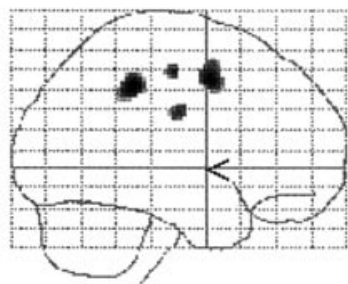

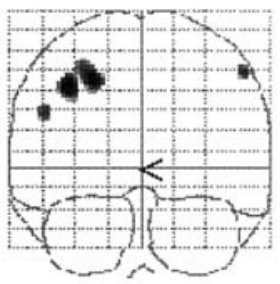

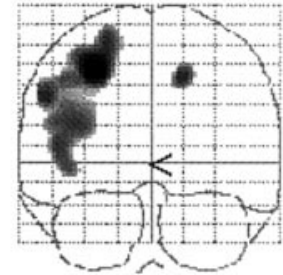

我地

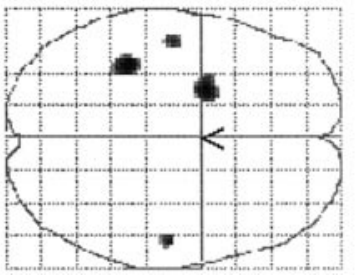

FIG. 3. Sagittal, coronal, and horizontal views of brain regions associated with encoding and retrieval of covert actions. (a) Differential activation during covert encoding enactment compared to verbal encoding. (b) Differential activation during retrieval following covert encoding enactment compared to retrieval following verbal encoding. The retrieval contrast was masked by the encoding contrast. The threshold for plotting activations in (a) was $\mathrm{P}<0.05$ corrected; the threshold for plotting activations in (b) was $\mathrm{P}<0.0005$ uncorrected (see text and Table 2 for inferential statistics).

specifically, as compared to the verbal condition, encoding by means of overt enactment and retrieval following overt encoding enactment were both associated with differential activation in several left hemisphere regions, notably parietal cortex and motor cortex (we refer to activations in the region of central sulcus as motor cortex; the resolution of the images did not allow differentiation between primary motor cortex and premotor cortex). This observation provides support for the suggestion that retrieval following encoding enactment can be based on motor information (Engelkamp, 1998; cf., Heil et al., 1999). A similar conclusion was reached in a recent PET study of retrieval-related activity following enacted encoding (Nilsson et al., 2000). In the latter study, retrieval following enacted encoding was associated with increased activity in right motor cortex $(x, y, z=12,-32,60)$. Activity in this region

$(x, y, z=14,-30,66)$ was increased during retrieval of enacted compared to verbal events in this study as well (data not shown), but this effect was not salient when the retrieval contrast was masked by the contrast between enacted and verbal encoding. This encoding contrast revealed increased activity in several left hemisphere regions that previously have been associated with arm and hand movements (see Figs. 2a and $4 a$ ). The tendency to a left lateralization of activations was expected as subjects executed the actions with their right $\mathrm{arm} / \mathrm{hand}$. It is hence possible that the present strategy of analyzing retrieval activity in light of encoding activity biased the retrieval results towards left hemisphere activations.

Encoding-retrieval overlap in motor areas was also observed for encoding and retrieval of imagined activi-
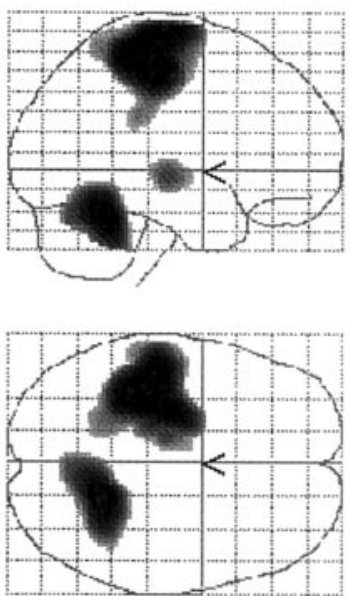

b
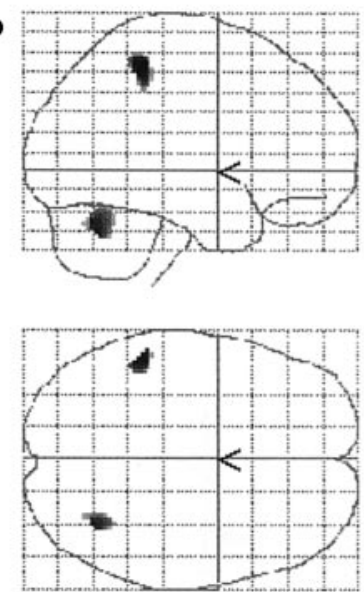
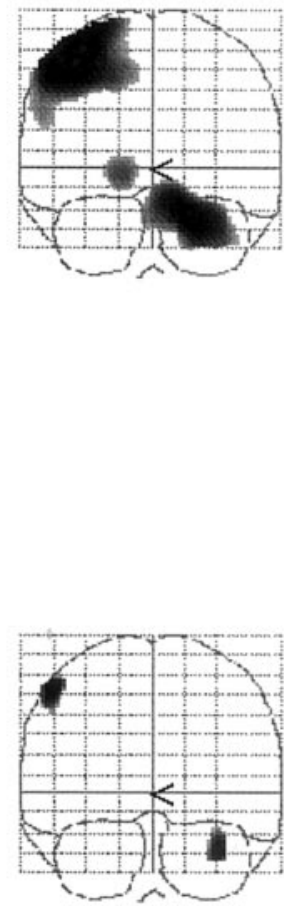

FIG. 4. Sagittal, coronal, and horizontal views of brain regions specifically associated with encoding and retrieval of overt actions. (a) Differential activation during overt encoding enactment compared to covert encoding enactment. (b) Differential activation during retrieval following overt encoding enactment compared to re trieval following covert encoding enactment. The retrieval contrast was masked by the encoding contrast. The threshold for plotting activations in (a) was $\mathrm{P}<0.05$ corrected; the threshold for plotting activations in (b) was $\mathrm{P}<0.005$ uncorrected (see text and Table 2 for inferential statistics). 
ties. Relative to verbal encoding, motor imagery was associated with increased activity in motor cortex. The observation of increased activity in motor cortex during imagined movement is in line with recent fMRI findings and reinforces thenotion that motor cortex function is not limited to movement execution (Lotze \& al., 1999). At retrieval, parts of motor/premotor cortex and inferior parietal cortex showed differential activity. The activation of motor cortex suggests that, just as for overt enactment, retrieval can be based on motor information following covert motor activity during encoding. Here it should be noted that the difference in memory performance between the imagery and verbal conditions did not reach significance. In principle, there need not be a conflict between an observation of lack of difference in memory performance between two conditions and an observation of differences in activation patterns. An encoding condition that involves analysis of shallow aspects of a message (e.g., if it is spoken in a male or female voice) will lead to poor retention on most episodic tests, but can still be associated with distinct activation at retrieval (cf., Nyberg et al., 1995). Nonetheless, in the present study there was a difference in memory performance between the imaginary and verbal conditions that was in the expected direction (significant at $\mathrm{P}<0.10$ ). This allows for the possibility that there was a relation between increased motor activity at retrieval and increased memory performance following imaginary motor encoding.

The results from the real and imaginary enactment conditions converged in showing increased activity in a ventral part of motor cortex during retrieval $(x, y, z=$ $-50,-12,28)$. As noted in the results section, the retrieval peak was considerably more ventral than the encoding peaks. The encoding peaks were near dorsal motor cortical regions that in previous studies have been associated with finger/shoulder movements (Fink et al., 1997), but the activations extended to include the ventral region. The reason why encoding-retrieval overlap was maximal for this ventral region is unclear, but it can be noted that increased activity in this part of motor cortex has previously been reported during motor imagery (Decety et al., 1994). Decety et al . (1994) related their finding to motor planning and programming, and it might be speculated that in the present study such processes were common to overt and covert encoding enactment as well as to retrieval following overt and covert encoding enactment. Another common activation during retrieval following overt and covert encoding enactment was in inferior parietal cortex ( $x$, $y, z=-46,-43,35)$. I ncreased activity in this region has previously been observed during finger/shoulder movements (Fink et al., 1997).

Notwithstanding the similarities in activation patterns for the real and imaginary enactment conditions, differences were also observed. These were especially pronounced during encoding, when real enactment more strongly activated contralateral somatosensory and motor cortex, midbrain, and ipsilateral cerebellum. This is in good agreement with comparisons of executed and imagined finger (Deiber et al., 1998) and hand (Lotze et al., 1999) movements. Differential activation of a left parietal and a right cerebellar region was observed when the real and imaginary retrieval conditions were contrasted. The parietal activation was more dorsal $(x, y, z=-51,-36,52)$ than the parietal region discussed in the above section (common to overt and covert enactment), and increased activity at a similar location was observed in the contrast be tween the overt enactment and verbal conditions (Table 2). Hence, this activation was consistently associated with the real enactment condition-regardless of whether the verbal or covert enactment condition was used as the reference condition.

The functional significance of this parietal activation remains unclear, but dorsal parietal regions have been associated with representation of somatosensory information (Hadjikhani and Roland, 1998). Possibly, then, the parietal activation reflects encoding and subsequent retrieval of somatosensory information. Left lateral parietal activation has also been associated with retrieval success (e.g., Konishi et al., 2000). Although the present peaks did not dosely overlap with those reported by Konishi et al., a retrieval success interpretation is consistent with the present results as retrieval was highest following real enactment during encoding.

In addition to left parietal cortex, right cerebellum was differentially active during encoding and retrieval of overt actions compared to the covert enactment condition. A plot of parameter estimates showed that activity was also higher in the real enactment condition compared to the verbal condition, but the effect was more pronounced when the overt and covert enactment conditions were contrasted. This can be related to previous findings of reduced cerebellar activity during imagined motor activity (Lotze et al., 1999). The site where maximal encoding-retrieval overlap was observed in the overt enactment condition $(x, y, z=-34$, $-64,-20)$ was different from the site where maximal cerebellar activation was observed when the real enactment encoding condition was contrasted with the overt and verbal encoding conditions (Figs. 2a and 4a; $x, y, z=18,-50,-18)$. Together with the data for motor cortex, this indicates that overlap in encodingretrieval activity is not necessarily found at the site where encoding activity is maximal. Finally, it should be noted that left-sided cerebellar activity was associated with retrieval (Fig. 1b). Thus, in keeping with the existence of crossed connections between frontal cortex and cerebellum, motor execution with the right hand was associated with left frontal and right cerebellar activation and episodic retrieval with right frontal and left cerebellar activation.

More generally, our observation of differential activation of traditional motor areas during explicit memory 
retrieval of specific event information extends findings that certain forms of implicit memory, such as motor sequence learning, are associated with motor brain areas (see Cabeza and Nyberg, 2000). It can also be related to findings indicating that motor cortex processes serial order information within the context of a motor task (Carpenter \& al., 1999). Collectively, these and other findings suggest that motor brain regions participate in certain types of nonmotor cognitive processes.

In summary, the present results are in agreement with the long-standing reactivation hypothesis that some of the brain regions that are active during perception/encoding of sensory information are reactivated during retrieval of the same information (Damasio, 1989; J ames, 1893). This principle seems to hold true not only for brain activity related to sensoryinduced perceptions but also for brain activity related to overt and covert motor execution.

\section{ACKNOWLEDG MENTS}

This work was supported by a grant from the Medical Research Council of Sweden (K 98-21X-12252-02B). L.N. was supported by the Swedish Council for Research in the Humanistic and Social Sciences (HSFR).

\section{REFERENCES}

Alvarez, P., and Squire, L. R. 1994. Memory consolidation and the medial temporal lobe: A simple network model. Proc. Natl. Acad. Sci. USA 91: 7041-7045.

Bäckman, L., and Nilsson, L.-G. 1984. Aging effects in free recall: An exception to the rule. Hum. Learn. 3: 53- 69.

Cabeza, R., and Nyberg, L. 2000. Imaging cognition II: En empirical review of 275 PET and fMRI studies. J . Cogn. Neurosci. 12: 1- 47.

Carpenter, A. F., Georgopoulos, A. P., and Pellizzer, G. 2000. Motor cortical encoding of serial order in a context-recall task. Science 283: 1752-1757.

Cohen, R. L., and Stewart, M. 1982. How to avoid developmental effects in free recall. Scand. J. Psychol. 23: 9-16.

Crammond, D. J. 1997. Motor imagery: never in your wildest dreams. Trends Neurosci. 20: 54-57.

Damasio, A. R. 1989. Time-locked multiregional retroactivation: A systems-level proposal for the neural substrates of recall and recognition. Cognition 33: 25- 62.

Decety, J ., Perani, D., J eannerod, M., Bettinardi, V., Tadary, B., Woods, R., Mazziotta, J. C., and Fazio, F. 1994. Mapping motor representations with positron emission tomography. Nature $\mathbf{3 7 1}$ : $600-602$.

Deiber, M.-P, Ibañez, V., Honda, M., Sadato, N., Raman, R., and Hallett, M. 1998. Cerebral processes related to visuomotor imagery and generation of simple finger movements studied with positron emission tomography. Neurol mage 7: 73- 85.

Engelkamp, J . 1998. Memory for Actions. Psychology Press, Hove, UK.

Fink, G. R., Frackowiak, R. S. J ., Pietrzyk, U., and Passingham, R. E. 1997. Multiple nonprimary motor areas in the human cortex. J . Neurophysiol. 77: 2164-2174.
Hadjikhani, N., and Roland, P. E. 1998. Cross-modal transfer of information between the tactile and the visual representations in the human brain: A positron emission tomographic study. J . Neurosci. 18: 1072-1084.

Heil, M., Rolke, B., Engelkamp, J ., Rösler, F., Özcan, M., and Henninghausen, E. 1999. Event-related brain potentials during recognition of ordinary and bizarre action phrases following verbal and subject-performed encoding conditions. Eur. J . Cogn. Psychol. 11: 261-280.

Herscovitch, P., Markham, J ., and Raichle, M. E. 1983. Brain blood flow measured with intravenous $\mathrm{H}_{2}{ }^{15} \mathrm{O}$. I. Theory and error analysis. J. Nucl. Med. 24: 782-789.

J ames, W. 1893. Principles of Psychology. Holt, New York.

Karlsson, T., Bäckman, L., Herlitz, A., Nilsson, L.-G., Winblad, B., and Österlind, P.-O. 1989. Memory impairment at different stages of Alzheimer's disease. Neuropsychologia 27: 737-742.

Köhler, S., Moscovitch, M., Winocur, G., Houle, S., and Mclntosh, A. R. 1998. Networks of domain-specific and general regions involved in episodic memory for spatial location and object identity. Neuropsychologia 36: 129-142.

Konishi, S., Wheeler, M. E., Donaldson, D. I., and Buckner, R. L. 2000. Neural correlates of episodic retrieval success. Neurol mage 12: 276-286.

Lotze, M., Montoya, P., Erb, M., Hülsmann, E., Flor, H., Klose, U., Birbaumer, N., and Grodd, W. 1999. Activation of cortical and cerebellar motor areas during executed and imagined hand movements: An fMRI study. J . Cogn. Neurosci. 11: 491-501.

McAndrews, M. P., and Milner, B. 1991. The frontal cortex and memory for temporal order. Neuropsychologia 29: 849-859.

Mimura, M., Komatsu, S., Kato, M., Yashimasu, H., Wakamatsu, N., and Kashima, H. 1998. Memory for subject-performed tasks in patients with Korsakoff syndrome. Cortex 34: 297-303.

Morris, C. D., Bransford, J. D., and Franks, J. J . 1979. Levels of processing versus transfer appropriate processing. J. Verbal Learn. Verbal Behav. 16: 519-533.

Nilsson, L.-G., Nyberg, L., Klingberg, T., Åberg, C., Persson, J ., and Roland, P. E. 2000. Activity in motor areas while remembering action events. NeuroReport 11: 2199-2201.

Nyberg, L., Habib, R., Mclntosh, A. R., and Tulving, E. 2000. Reactivation of encoding-related brain activity during memory retrieval. Proc. Natl. Acad. Sci. USA 97: 11120-11124.

Nyberg, L., and Nilsson, L.-G. 1995. The role of enactment in implicit and explicit memory. Psycho. Res. 57: 215-219.

Nyberg, L., Tulving, E., Habib, R., Nilsson, L.-G., Kapur, S., Houle, S., Cabeza, R., and Mcl ntosh, A. R. 1995. Functional brain maps of retrieval mode and recovery of episodic information. NeuroReport 7: $249-252$.

Persson, J ., and Nyberg, L. 2000. Conjunction analysis of cortical activations common to encoding and retrieval. Microsc. Res. Tech. 51: $39-44$.

Price, C. J ., and Friston, K. J . 1997. Cognitive conjunction: A new approach to brain activation experiments. Neurol mage 5: 261-270.

Talairach, J ., and Tournoux, P. 1988. Co-Planar Stereotaxic Atlas of the Human Brain. Thieme, New York.

Wienhard, K., Dahlbom, M., Eriksson, L., Michel, C., Bruckbauer, T., Pietrzyk, U., and Heiss, W.-D. 1994. The ECAT EXACT HR: Performance of a new high resolution positron scanner. J . Comput. Assist. Tomogr. 18: 110-118.

Wheeler, M. E., Petersen, S. E., and Buckner, R. L. 2000. Memory's echo: Vivid remembering reactivates sensory-specific cortex. Proc. Natl. Acad. Sci. USA 97: 11125-11129. 